\title{
Series de televisión y percepción de prejuicios acerca de la figura del inmigrante. Un estudio con universitarios
}

Immigranteei buruzko telebista saioak eta aurreiritzien pertzepzioa. Ikerketa unibertsitateko ikasleekin

T.V. Series and perception of prejudices towards the figure of the immigrant. A study with university students

Martin Yael Santana Mejía`, Juan Ignacio Martínez de Morentin, Concepción Medrano Samaniego

$\mathrm{UPV} / \mathrm{EHU}$

RESUMEN: La presente investigación analiza prejuicios en personajes inmigrantes que perciben jóvenes universitarios en las series que ven. Este es un estudio preliminar que cuenta con una muestra de 70 alumnos en los grados de educación infantil y comunicación audiovisual. Para ello se ha utilizado el cuestionario de percepción de prejuicios de la diversidad cultural en series de tv creado "ad hoc» para este estudio. Se han hallado datos significativos sobre prejuicios percibidos en función a la titulación del alumnado, formato de la serie y el origen de producción. En vista de los datos encontrados podemos decir que los prejuicios percibidos varían según características de las series y del espectador.

PALABRAS CLAVE: Series de televisión; Prejuicios; Inmigrante; Formato; Producción.

LABURPENA: Ikerketa honek unibertsitateko gazteek ikusten dituzten telebista saioetan immigranteen inguruan antzematen dituzten aurreiritziak aztertzen ditu. Aurretiko ikerketa da, Haur Hezkuntza eta Ikus-entzunezko Komunikazioa graduetako 70 ikasleren laginak biltzen dituena. Horretarako, ikerketa honetarako ad hoc sortu den galdetegia erabili da, zehazki, hainbat telebista saiotako kultur aniztasunaren aurreiritzien pertzepzioari buruzko galdetegia. Oso datu esanguratsuak lortu dira antzemandako aurreiritziei buruz, ikasleen titulazioaren arabera, saioaren formatuaren arabera eta ekoizpenaren jatorriaren arabera. Horiek horrela, 
esan dezakegu antzemandako aurreiritziak aldatu egiten direla saioen eta ikus-entzuleen ezaugarrien arabera.

HITZ-GAKOAK: Telebista saioak; aurreiritziak; immigrantea; formatua; ekoizpena.

\begin{abstract}
The present investigation analyzes prejudices towards to immigrant characters that are perceived by university students in the TV series that they watch. This is a preliminary study, which has a sample of 70 students in the grades of early childhood education and audiovisual communication. For this purpose, was used the questionnaire on the perception of cultural diversity prejudices in TV series created "ad hoc». We have found significant data on perceived prejudices based on the students' qualifications, format of the series and the origin of production. Regarding the found data, we had determinate that the perceived prejudices vary according to the characteristics of the series and the viewer.
\end{abstract}

KEYWORDS: T.V. series; Prejudices; Immigrant; Format; Production.

^ Correspondencia a: Martin Yael Santana Mejía. UPV/EHU. Facultad de Psicología, avda. de Tolosa, 70 (20018 San Sebastián).martinyaelsantana@gmail.com http://orcid.org/0000-0003-0766-2747

Cómo citar: Santana Mejía, Martin Yael; Martínez de Morentin, Juan Ignacio; Medrano Samaniego, Concepción. 2018. Series de televisión y percepción de prejuicios acerca de la figura del inmigrante. Un estudio con universitarios. Zer. 23(45). 203-225. https://doi.org/10.1387/zer.20030

Recibido: 3 julio 2018; aceptado: 9 noviembre 2018

1137-1102 y 1989-631X / C 2018 UPV/EHU 


\section{Introducción}

Los medios de comunicación tienen un papel importante en la percepción que posee la juventud sobre la diversidad cultural. En este sentido, uno de los agentes de socialización, junto con la familia y los iguales, que es preciso tener en cuenta de acuerdo a las investigaciones previas, es el contenido televisivo, por ser uno de los más consumidos y al que cada vez se facilita más el acceso a través de diferentes plataformas (López Vidales, González Aldea y Medina de la Viña, 2011). Evidentemente como afirman Martín, Muntada, Gotzens, Cladellas y Descallar (2015) «La televisión es un arma poderosa que ha desplazado, en muchos casos, a la lectura y a otras actividades lúdicas» (p. 28).

De acuerdo al estado del arte, la televisión es capaz de modelar nuestras actitudes e influir en ellas y parece estar haciéndolo de manera constante (Medrano y Palacios 2008; Medrano, Martínez de Morentin y Pindado, 2014). Este medio de comunicación contribuye a la construcción de la imagen social y la percepción que pueda tener una persona sobre el mundo (Gerbner, 1996; Signorielli, Morgan, Gross y Gerbner, 1996). Esta percepción colectiva que se crea con lo que se transmite a través de la ficción televisiva entre otras variables puede forjar las actitudes de toda una generación (Montero, 2005).

En este contexto, de acuerdo a distintas investigaciones la juventud consume y exige más ficción en la programación televisiva (López Vidales, Medina de la Viña y González Aldea, 2013). Así mismo, existe evidencia empírica respecto a que, aproximadamente, la mitad de los jóvenes que perciben la inmigración en contenidos televisivos lo hace en series de televisión, y en éste y los demás medios el exogrupo es relacionado con temas contraproducentes para la sociedad (Prats, De Régil, Lobarte, Higueras y Santamaría 2005).

De acuerdo con Bermejo y Núñez (2008) y Moreno y Rodríguez (2016) las series de televisión son los contenidos que mayor consumo presentan, por ello es importante estudiar los prejuicios que perciben los jóvenes espectadores de los inmigrantes en dichos contenidos, dado que los mismos pueden estar influyendo en las actitudes o prejuicios acerca de la inmigración que los jóvenes puedan desarrollar a lo largo de su vida. Si bien es cierto que los medios tienen mucha influencia en el ámbito social, éstos no deciden por las personas, pero sí pueden manejar los temas a los que tienen acceso las personas en su diario vivir (Díaz, 2004). 


\section{Marco teórico}

\subsection{TeleVisión Y PERCEPCiÓN DE PREJUiCIOS EN LA INMIGRACIÓN}

El prejuicio es una concepción que puede ser negativa o positiva hacia una persona por pertenecer a un determinado grupo, basándose o no en alguna experiencia previa (Allport, Malfé y Verón, 1968; Igartua y Frutos, 2016). No obstante, en la actualidad, cuando nos referimos a prejuicios se señala una concepción negativa, y estas actitudes en los medios de comunicación están consideradas como una forma de racismo (Van Dijk, 2005).

Gran parte de la investigación disponible acerca de la figura del inmigrante en los medios está centrada en la prensa. De acuerdo con Gálvez (2009) en la prensa el tema de la inmigración se presenta de forma negativa. Esto es consecuencia de la forma sesgada en la que se presentan las diferentes etnias en las noticias (Igartua, 2013). Por otro lado, Giró y Jarque (2006) explica que la prensa escrita enfoca sus noticias para fomentar la integración de la inmigración, sin embargo la mayor parte de los editoriales plantean controlar la inmigración lo que le hace ver como un fenómeno «peligroso». En esta línea Galán (2006) llegó a la conclusión que este tratamiento de la prensa es muy rico en recursos dramáticos, y estos recursos son los que utilizan los guionistas para la creación de personajes de ficción televisiva; de forma que, el prejuicio que se percibe tanto en la ficción televisiva como en la prensa sigue una misma tendencia. Una revisión reciente de Eberl et. al (2018) sobre el tema indica que:

A pesar de que es más probable que la audiencia esté más expuesta a contenidos de entretenimiento, series o películas, que a noticias en los medios. Ignoramos el discurso social del entretenimiento sobre la inmigración y los grupos de inmigrantes, descuidamos una parte de la realidad de los medios, que una gran parte del público europeo usa exclusivamente (p. 128).

Hay que destacar el hecho de que Estados Unidos es uno de los países donde empiezan a tomarse en cuenta las minorías respecto a la ficción, ya sea en cine o televisión, debido a que fueron pioneros en cuanto a la producción de contenidos audiovisuales, lo que logra que las representaciones raciales en su ficción formen parte de la creación de su identidad social (Lacalle, 2008). En este contexto se han realizado investigaciones respecto al tema de gran relevancia, las cuales han arrojado resultados similares a través del tiempo, entre los que se destacan la infrarrepresentación de la inmigración en las series, los estereotipos negativos en los personajes inmigrantes, caracterizados por empleos bajos, roles de poca importancia, bajo nivel educativo, violencia y diferentes aspectos enmarcados como prejuicios (Harwood y Anderson 2002; Mastro y Greenberg, 2000; Mastro y Behm-Morawitz 2005; Monk-Turner, Heiserman, Johnson, Cotton y Jackson 2010). 
Ramos (2014) analizó el contenido de ficción televisiva en la franja prime time en el cual se halló una imagen del inmigrante sesgada y negativamente estereotipada, donde la mayoría desempeña roles de villanos y en general son personajes violentos El fenómeno también se ha estudiado en la última década desde el enfoque del framing y el análisis de discurso, que son técnicas diferentes a las utilizadas por los autores citados anteriormente. Aunque los resultados son similares encontrando una imagen del inmigrante negativa y estereotipada (Adams 2018; Arellano, 2017).

En efecto, la imagen que se transmite en los mass media acerca del exogrupo trae consigo diferentes prejuicios incluyendo colocarlos como villanos/antagonistas (Ramos, Igartua, Frutos, Barrios, Mohedano y Naval, 2014). Asimismo, esta imagen va acompañada de un discurso social que continuamente se ve reforzado de manera explícita en programas televisivos, series de televisión y películas (Benavides y Retis, 2005; Galán, 2009; Van Dijk, 2007). Lacalle (2008) en su estudio halló que dentro de algunas características generales de 30 series que estudió, los personajes inmigrantes están mejor representados en las series dramáticas que en las comedias. Asimismo también se les asocia con estatus de ilegalidad y tener ocupaciones de bajo nivel. El 80\% de personajes inmigrantes analizados están representados como víctimas en la trama, víctimas de personajes españoles a nivel de explotación y/o violencia.

También, algunos autores señalan que existen series de producción española que contienen prejuicios en personajes inmigrantes, donde, habitualmente, se les muestra como embaucadores, seductores y extrovertidos igualmente aparecen en roles sin importancia y siendo marginados ligados a tramas violentas (Galán, 2006; 2009; Igartua, Barrios y Ortega, 2012; Ruiz, Ferrer, Obradors, Pujadas, y Pérez, 2007). Además, cuando se trata de diversidad cultural en series de televisión o ficción televisiva los inmigrantes tienen cierta tendencia a ser antagonistas/villanos (Igartua, Marcos, Alvidrez y Piñeiro 2013). Cabe señalar que lo que perciben los espectadores en las series de ficción provoca cambios en las actitudes de estos, este componente de la influencia que ejercen los contenidos sobre los individuos es conocido como persuasión narrativa, el cual es bastante novedoso y no poseemos, a penas, investigaciones en este ámbito. (Igartua, 2007). En los cambios de actitud que describe la persuasión narrativa se indica la existencia de una amplia incidencia de la identificación con los personajes entre otros procesos mediadores (Cohen, 2001; Igartua, 2008; Igartua y Páez, 1998; Moyer-Gusé, 2008). Está demostrado que el público se identifica con mayor facilidad con un personaje de su nacionalidad o grupo social (Cohen, 2001; Ugalde, Medrano y Aierbe 2012). Estos cambios de actitud propiciados por la persuasión narrativa han sido evidenciados en estudios realizados en Estados Unidos, en función de cómo se representa la diversidad cultural en la ficción (Muñiz, Serrano, Aguilera y Rodríguez, 2013). 


\subsection{Formatos televisivos}

Las series de televisión poseen una taxonomía referida a su clasificación. Por lo tanto, la ficción televisiva como género abarca lo que es una miniserie, una serie y un telefilme. Dentro de las categorías, anteriormente, mencionadas hay que destacar los dramas y las comedias con sus respectivos formatos que funcionan como subcategorías (Soap opera, telenovela, drama, sitcom, dramedy, etc.) en los cuales se clasifican estos contenidos (Carrasco, 2010).

De acuerdo a los datos hallados en la investigación previa, los géneros y formatos televisivos tienen influencia en lo que el espectador pueda percibir respecto a la diversidad cultural (Hoekstra, Stos, Swendson y Hoekstra, 2012). Atendiendo a estas consideraciones existen estudios que establecen que en las series dramáticas tienen múltiples prejuicios hacia una determinada minoría, a pesar de que están buscan tener una visión más realista (Galán, 2006). En efecto, esta línea de crear ficción realista dentro del formato drama es una fórmula que le da a la ficción televisiva historias con situaciones y tramas creíbles y, al mismo tiempo, con valores y tendencias sociales de actualidad, de ahí que el público pueda entrar contacto con éstas y percibir diferentes prejuicios (Castro, 2002).

Cabe destacar que las series de comedia no provocan respuestas reflexivas sino más bien de disfrute, esto no genera mucha empatía hacia los personajes que formen parte de alguna trama. En cambio, la ficción que contiene drama puede provocar otro tipo de efecto en los espectadores que la prefieren ya que éstos buscan contemplar verosimilitud en lo que eligen para entretenerse con la necesidad de auto-reflexión (Oliver y Raney, 2011).

En esta línea y, teniendo en cuenta la revisión del estado del arte, esta investigación aborda como objeto de estudio la percepción de prejuicios hacia la inmigración en series de televisión en función del formato televisivo que prefieren los estudiantes universitarios, al igual que el origen de producción de la serie. Igartua y Frutos (2016) sugieren que en el futuro deben existir más investigaciones donde se aborde este tópico, y de esta forma contrastar si los formatos narrativos que ofrece el entretenimiento mediático estimulan en mayor o menor medida la reflexión en el espectador. Asimismo se toma en cuenta en la investigación de Lacalle (2008) que los inmigrantes están mejor representados en los dramas que en las comedias.

\subsection{INVESTIGACIONES CON UNIVERSITARIOS}

A pesar de que la investigación académica sobre la televisión es bastante extensa, las investigaciones con alumnado universitario son bastante escasas (Martínez-Serrano, 
2014), y cabe señalar que la mayoría de estas investigaciones con minorías están basadas en los medios de comunicación de corte noticioso (Ramos, 2014), y en su mayor parte están enfocadas en la percepción negativa que se transmite del exogrupo (Park, 2012).

Existen estudios actuales acerca de series de televisión y la diversidad cultural enfocados desde la educación-entretenimiento para propiciar cambios positivos en la percepción de prejuicios hacia el exogrupo. Así Müller (2009) halló datos que demuestran que el contacto de individuos de culturas diferentes puede fomentarse a través de las series de TV. En esta línea, Igartua y Ramos (2013) plantearon una intervención en estudiantes universitarios con largometrajes para favorecer las actitudes hacia los inmigrantes. En cuanto a consumo de ficción de estudiantes universitarios Martínez-Serrano (2014) halló que la mayor parte del tiempo que los estudiantes dedican a esta actividad, éstos consumen series locales en primer lugar y, en segundo lugar, series extranjeras. En el trabajo que se presenta se trata de analizar qué tipo de prejuicio perciben los sujetos dependiendo del origen de producción (local o extranjera) de la serie.

Igartua y Frutos (2016) en un trabajo con estudiantes universitarios afirman que «es más efectiva una estrategia basada en fomentar la empatía hacia los inmigrantes que mostrar, con un tono desenfadado o cómico, situaciones de contacto íntimo de carácter intergrupal» (p. 57). Por lo que la presente investigación, se propone analizar los formatos de las series y los prejuicios verificando qué prejuicios pueden ser percibidos si es comedia o drama.

En otros estudios de diferentes modalidades realizados también con alumnado universitario se ha establecido un incremento de la matriculación en las profesiones que aparecen en series de ficción, poniendo en evidencia la influencia que tiene la ficción en las actitudes e intereses de los jóvenes (Montero, 2006).

En definitiva, la investigación previa sobre la inmigración en la ficción que consumen los jóvenes tiene aportes significativos; asimismo, genera investigación para crear y concienciar a la juventud y lograr que tengan una mejor competencia mediática. Una competencia necesaria, en la actualidad, ya que vivimos en una sociedad multipantalla.

\section{Objetivos}

El principal objetivo de esta investigación es conocer los prejuicios hacia el inmigrante que percibe el alumnado universitario en las series de televisión que más le gustan. A partir de un objetivo general, y los objetivos específicos que son los siguientes: 
1. Conocer la percepción de algunas variables conceptualizadas como prejuicios en el personaje inmigrante que son: rol narrativo, violencia como agresor, violencia como víctima, hábitos nocivos y temas de conversación. En las series de tv favoritas elegidas por la muestra en función de:

1.1. El tipo de titulación del alumnado de primer año de: Educación infantil y Comunicación audiovisual.

1.2. El formato de la serie.

1.3. El origen de producción de la serie.

\section{Método}

Se trata de un estudio piloto con un diseño descriptivo, puesto que éste es el diseño que se ajusta a las necesidades de esta investigación. No se manipulan las variables.

\subsection{Muestras}

La muestra para este estudio es de conveniencia y se recolectaron datos de 70 (58 chicas y 12 chicos) estudiantes de la Universidad del País Vasco de dos carreras universitarias que son: Comunicación y Educación infantil, y con el fin de establecer comparaciones en estas dos áreas de las ciencias sociales. Ya que el alumnado de comunicación puede tener una perspectiva diferente sobre los personajes inmigrantes en las series que los alumnos de magisterio.

\subsection{INSTRUMENTOS}

El instrumento utilizado para este estudio es el Cuestionario de Percepción de prejuicios de la diversidad cultural en series de tv (PPDC-STV), creado, "ad hoc» para los fines de esta investigación. Los ítems de este cuestionario se han elaborado partiendo de los códigos del análisis de contenido utilizados por Igartua, Barrios y Ortega (2012) en su estudio "Analysis of the Image of Immigration in Prime Time Televisión Fiction» en el cual se encontraron indicadores de cómo se muestra la diversidad cultural en la series de tv en la actualidad. Se llevó a cabo un análisis de fiabilidad mediante el coeficiente de acuerdo observado (OA) y Pi de Scott para variables cualitativas. Con una media de 55 variables consideradas el $\mathrm{OA}=0,86$, y $\pi=0,75$ unos valores muy aceptables.

El cuestionario cuenta con 9 dimensiones las cuales pretenden medir lo que percibe la muestra acerca de los personajes inmigrantes en diferentes aspectos sociales 
que involucran prejuicios y con la frecuencia que el espectador los recibe dentro de la ficción televisiva. Estas dimensiones son:

—Dimensión rol Narrativo (Ítem 1).

-Dimensión educativa del personaje (Ítem 2).

—Dimensión laboral del personaje (Ítem 3).

-Dimensión económica del personaje (Ítem 4).

—Dimensión de aspectos de violencia del personaje (Ítem 5 y 6).

—Dimensión de hábitos nocivos del personaje (Ítem 7).

—Dimensión conversacional de personaje (Ítem 8).

—Dimensión de cualidad personal del personaje (Ítem 9).

Para este trabajo se han utilizado únicamente 4 dimensiones debido a su amplitud y son: rol narrativo, la violencia del personaje, hábitos nocivos y dimensión conversacional del personaje.

El análisis de fiabilidad fue realizado en las dimensiones que fueron tomadas en cuenta para su utilización y creación en el instrumento son: la dimensión de aspectos de violencia con un alpha de .758 , dimensión de hábitos nocivos con un alpha .822 de y dimensión conversacional del personaje con un alpha de .792. Se ha obviado la dimensión de cualidad personal del personaje para esta investigación ya que ésta arrojó una fiabilidad -.089 debido al planteamiento utilizado con el ítem y sus opciones de respuesta.

Los ítems de este cuestionario son de tipo Likert con opciones de respuestas múltiples según requiere el carácter del ítem. Fue elaborado y utilizado en versión digital con la finalidad de optimizar su cumplimentación y recogida así como el manejo de los datos.

\subsection{Procedimiento}

Se contactaron profesores del área de educación y comunicación en la Universidad del País Vasco para verificar la disponibilidad de sus alumnos en la participación de este estudio piloto. Previamente a la prueba se les explico que no era obligatoria. Una vez aprobada la solicitud por los profesores responsables se acordaron fechas y horarios de recogida.

Esta investigación fue aprobada por el comité de Ética de la UPV/EHU, para la recogida de datos con seres humanos, dentro de la línea de investigación «Valores, socialización y medios de comunicación». Se ha salvaguardado la identidad y la integridad psicológica de los participantes, y por confidencialidad, no constará en los cuestionarios la identidad de los sujetos. 
Una vez en el aula, se procedió a explicar oralmente la forma correcta de la cumplimentación del instrumento y se aplicaron los cuestionarios PPDC-STV, donde las y los sujetos suministrarán los datos sobre sus series de TV favorita y el personaje inmigrante que aparezca en esta. El tiempo de cumplimentación del instrumento es de 20-25 minutos.

\section{Resultados}

A continuación se muestran los resultados obtenidos según los objetivos trazados de este estudio.

Objetivo 1.1: Conocer la percepción de algunas variables conceptualizadas como prejuicios como son el rol narrativo, violencia como agresor, violencia como víctima, hábitos nocivos y temas de conversación en el personaje inmigrante. En función de la titulación en el alumnado de primer año de: Educación infantil y Comunicación audiovisual

La tabla 1 muestra las diferencias significativas entre ambos grupos respecto al rol de secundario no protagónico del personaje inmigrante. Los estudiantes de comunicación percibieron más al personaje inmigrante en el rol secundario no protagónico que los de educación infantil.

\section{TABLA 1}

Rol narrativo en función de la titulación del alumnado

\begin{tabular}{|l|l|c|c|c|c|c|}
\hline \multicolumn{1}{|c|}{ Variable } & \multicolumn{1}{|c|}{ Grados } & N & Media & DT & t & p \\
\hline \multirow{2}{*}{ Rol Protagonista } & Educ. infantil & 35 & 2.80 & 1.30 & \multirow{2}{*}{1.42} & \multirow{2}{*}{1.00} \\
& Comunicación & 35 & 2.80 & 1.10 & & \\
\hline \multirow{2}{*}{ Rol Antagonista } & Educ. infantil & 35 & 2.26 & 1.14 & \multirow{2}{*}{.458} & .514 \\
& Comunicación & 35 & 2.09 & 1.04 & & \\
\hline \multirow{2}{*}{ Rol Secundario protagónico } & Educ. infantil & 35 & 3.37 & 1.06 & \multirow{2}{*}{.173} & \multirow{2}{*}{.832} \\
& Comunicación & 35 & 3.31 & 1.18 & & \\
\hline \multirow{2}{*}{ Rol secundario no protagónico } & Educ. infantil & 35 & 2.09 & 1.09 & \multirow{2}{*}{.149} & \multirow{2}{*}{.016} \\
& Comunicación & 35 & 2.69 & 1.13 & & \\
\hline
\end{tabular}

En lo que respecta a las variables violencia como agresor y violencia como víctima percibida del personaje inmigrante por ambos grupos, tomando en cuenta su titulación, no se encontraron diferencias estadísticamente significativas entre ellos. 
En cuanto a la variable de hábitos nocivos se encontraron diferencias significativas de medias, en el hábito nocivo de fumador, esto puede se puede observar en la tabla 2. Lo que indica que el alumnado de comunicación ha percibido más al inmigrante como fumador que el alumnado de educación infantil.

TABLA 2

Hábitos nocivos en función de la titulación del alumnado

\begin{tabular}{|l|l|c|c|c|c|c|}
\hline \multicolumn{1}{|c|}{ Variable } & \multicolumn{1}{|c|}{ Grados } & $\mathrm{N}$ & Media & $\mathrm{DT}$ & $\mathrm{t}$ & $\mathrm{p}$ \\
\hline \multirow{2}{*}{ Fumador } & Educ. infantil & 35 & 1.11 & .471 & \multirow{2}{*}{20.6} & \multirow{2}{*}{.017} \\
& Comunicación & 35 & 1.63 & 1.14 & & \\
\hline \multirow{2}{*}{ Alcoholismo } & Educ. infantil & 35 & 1.54 & .980 & \multirow{2}{*}{.830} & .155 \\
& Comunicación & 35 & 1.91 & 1.17 & & \\
\hline \multirow{2}{*}{ Adicción a Fármacos } & Educ. infantil & 35 & 1.23 & .690 & \multirow{2}{*}{3.28} & -.200 \\
& Comunicación & 35 & 1.43 & 1.00 & & \\
\hline \multirow{2}{*}{ Adicción a sustancias ilegales } & Educ. infantil & 35 & 1.14 & .430 & \multirow{2}{*}{9.22} & -.286 \\
& Comunicación & 35 & 1.43 & 1.09 & & \\
\hline
\end{tabular}

Respecto a los temas de conversación recurrentes del personaje inmigrante que percibió la muestra, no se encontraron diferencias estadísticamente significativas entre el alumnado de las dos titulaciones diferentes.

Objetivo 1.2: Conocer la percepción de algunas variables conceptualizadas como prejuicios como son el rol narrativo, violencia como agresor, violencia como víctima, hábitos nocivos y temas de conversación en el personaje inmigrante de las series de tv favoritas, en función del formato de la serie.

Se realizaron pruebas $\mathrm{t}$ de student para evaluar si las medias tienen diferencias estadísticamente significativas respecto al formato de las series que prefiere la muestra.

En el caso de la variable de rol narrativo del personaje inmigrante, la prueba t muestra que se encontraron diferencias significativas en dos de los roles de esta variable como se observa en la tabla 3. Por lo que los roles de protagonista y de antagonista fueron más percibidos en los dramas que en las comedias. 
TABLA 3

Rol narrativo percibido en función del formato de la serie

\begin{tabular}{|c|c|c|c|c|c|c|}
\hline Variable & Formato & $\mathrm{N}$ & Media & DT & $\mathrm{t}$ & $\mathrm{p}$ \\
\hline Rol Protagonista & $\begin{array}{l}\text { Drama } \\
\text { Comedia }\end{array}$ & $\begin{array}{l}31 \\
39\end{array}$ & $\begin{array}{l}3.23 \\
2.46\end{array}$ & $\begin{array}{l}1.23 \\
1.07\end{array}$ & 2.77 & .007 \\
\hline Rol Antagonista & $\begin{array}{l}\text { Drama } \\
\text { Comedia }\end{array}$ & $\begin{array}{l}31 \\
39\end{array}$ & $\begin{array}{l}2.55 \\
1.87\end{array}$ & $\begin{array}{l}1.15 \\
.951\end{array}$ & 2.69 & .009 \\
\hline Rol Secundario protagónico & $\begin{array}{l}\text { Drama } \\
\text { Comedia }\end{array}$ & $\begin{array}{l}31 \\
39\end{array}$ & $\begin{array}{l}3.52 \\
3.21\end{array}$ & $\begin{array}{l}1.02 \\
1.17\end{array}$ & 1.16 & .249 \\
\hline Rol Secundario no protagónico & $\begin{array}{l}\text { Drama } \\
\text { Comedia }\end{array}$ & $\begin{array}{l}31 \\
39\end{array}$ & $\begin{array}{l}2.19 \\
2.49\end{array}$ & $\begin{array}{l}1.19 \\
1.12\end{array}$ & -1.05 & .294 \\
\hline
\end{tabular}

En el caso de la variable de violencia percibiendo al personaje inmigrante como agresor en función del formato de la serie (drama-comedia), se encontraron diferencias significativas en todas medias así como de los tipos de violencia exceptuado la violencia sexual; como se observa en la tabla 4. De acuerdo a los resultados obtenidos se puede decir que en el caso del formato drama la muestra percibió en el personaje inmigrante que éste utiliza más la violencia física, la intimidación, el daño a la propiedad y la violencia verbal.

TABLA 4

Violencia como agresor percibida en función del formato de la serie

\begin{tabular}{|c|c|c|c|c|c|c|}
\hline Variable & Formato & $\mathbf{N}$ & Media & DT & $\mathrm{t}$ & $\mathrm{p}$ \\
\hline Violencia física/agresor & $\begin{array}{l}\text { Drama } \\
\text { Comedia }\end{array}$ & $\begin{array}{l}31 \\
39\end{array}$ & $\begin{array}{l}2.84 \\
1.33\end{array}$ & $\begin{array}{l}1.18 \\
.701\end{array}$ & 6.61 & .000 \\
\hline Intimidación/agresor & $\begin{array}{l}\text { Drama } \\
\text { Comedia }\end{array}$ & $\begin{array}{l}31 \\
39\end{array}$ & $\begin{array}{l}3.13 \\
1.49\end{array}$ & $\begin{array}{l}1.28 \\
.854\end{array}$ & 6.40 & .000 \\
\hline Daño a la propiedad/agresor & $\begin{array}{l}\text { Drama } \\
\text { Comedia }\end{array}$ & $\begin{array}{l}31 \\
39\end{array}$ & $\begin{array}{l}2.29 \\
1.21\end{array}$ & $\begin{array}{l}1.10 \\
.570\end{array}$ & 5.32 & .000 \\
\hline Violencia verbal/agresor & $\begin{array}{l}\text { Drama } \\
\text { Comedia }\end{array}$ & $\begin{array}{l}31 \\
39\end{array}$ & $\begin{array}{l}2.90 \\
1.49\end{array}$ & $\begin{array}{l}1.27 \\
.914\end{array}$ & 5.41 & .000 \\
\hline Violencia sexual/agresor & $\begin{array}{l}\text { Drama } \\
\text { Comedia }\end{array}$ & $\begin{array}{l}31 \\
39\end{array}$ & $\begin{array}{l}1.55 \\
1.10\end{array}$ & $\begin{array}{l}1.20 \\
.502\end{array}$ & 2.09 & .061 \\
\hline
\end{tabular}

Se encontraron diferencias significativas en la variable violencia percibiendo al personaje inmigrante como víctima en el ítem de violencia fisica, dichas diferen- 
cias se pueden observar en la tabla 5. Es decir, en el formato drama se percibe más al personaje inmigrante como víctima de violencia física que como al resto de violencias como víctima.

TABLA 5

Violencia como víctima percibida en función del formato

\begin{tabular}{|l|l|c|c|c|c|c|}
\hline \multicolumn{1}{|c|}{ Variable } & \multicolumn{1}{|c|}{ Formato } & $\mathrm{N}$ & Media & $\mathrm{DT}$ & $\mathrm{t}$ & $\mathrm{p}$ \\
\hline \multirow{2}{*}{ Violencia fisica/víctima } & Drama & 31 & 2.52 & 1.02 & \multirow{2}{*}{2.67} & \multirow{2}{*}{.009} \\
& Comedia & 39 & 1.82 & 1.12 & & \\
\hline \multirow{2}{*}{ Intimidación/víctima } & Drama & 31 & 2.71 & .973 & \multirow{2}{*}{.648} & \multirow{2}{*}{.520} \\
\hline \multirow{2}{*}{ Daño a la propiedad/víctima } & Comedia & 39 & 2.51 & 1.55 & & \\
\hline \multirow{2}{*}{ Violencia verbal/víctima } & Drama & 31 & 1.87 & 1.08 & \multirow{2}{*}{.65} & \multirow{2}{*}{.103} \\
& Comedia & 39 & 1.49 & .854 & & \\
\hline \multirow{2}{*}{ Violencia sexual/víctima } & Drama & 31 & 2.74 & 1.12 & \multirow{2}{*}{.289} & .774 \\
& Comedia & 39 & 2.85 & 1.74 & & \\
\hline
\end{tabular}

Respecto a la variable de hábitos nocivos percibidos en el personaje inmigrante no se han encontrado diferencias significativas en las medias de ambos grupos (drama-comedia), esto puede observarse en la tabla 6.

TABLA 6

Percepción de hábitos nocivos en función del formato de la serie

\begin{tabular}{|l|l|c|c|c|c|c|}
\hline \multicolumn{1}{|c|}{ Variable } & \multicolumn{1}{|c|}{ Formato } & N & Media & DT & T & p \\
\hline \multirow{2}{*}{ Fumador } & Drama & 31 & 1.45 & 1.15 & \multirow{2}{*}{.659} & .512 \\
& Comedia & 39 & 1.31 & .655 & & \\
\hline \multirow{2}{*}{ Alcoholismo } & Drama & 31 & 1.81 & 1.19 & .531 & .597 \\
& Comedia & 39 & 1.67 & 1.00 & & \\
\hline \multirow{2}{*}{ Adicción a fármacos } & Drama & 31 & 1.42 & 1.05 & \multirow{2}{*}{.782} & .437 \\
& Comedia & 39 & 1.26 & .677 & & \\
\hline \multirow{2}{*}{ Adicción a sustancias ilegales } & Drama & 31 & 1.48 & 1.15 & \multirow{2}{*}{.179} & .077 \\
& Comedia & 39 & 1.13 & .409 & & \\
\hline
\end{tabular}

De acuerdo con la variable de temas conversación percibidos del personaje inmigrante se encontraron diferencias significativas en el tema de la violencia en el 
caso del formato drama, como se puede ver en la tabla 7 , lo que indica que los que prefieren el drama perciben que el personaje inmigrante habla más de este tema que los que prefieren en la comedia.

También se aprecia en este resultado que en el formato comedia se percibe que el personaje inmigrante utiliza más los temas de conversación recurrentes como el amor, medio ambiente y la inmigración.

TABLA 7

Temas de conversación recurrente en función del formato de la serie

\begin{tabular}{|c|c|c|c|c|c|c|}
\hline Variable & Formato & $\mathbf{N}$ & Media & DT & $\mathrm{t}$ & $\mathbf{P}$ \\
\hline Amor & $\begin{array}{l}\text { Drama } \\
\text { Comedia }\end{array}$ & $\begin{array}{l}31 \\
39\end{array}$ & $\begin{array}{l}2.90 \\
3.46\end{array}$ & $\begin{array}{l}1.24 \\
1.02\end{array}$ & -2.05 & .043 \\
\hline Violencia & $\begin{array}{l}\text { Drama } \\
\text { Comedia }\end{array}$ & $\begin{array}{l}31 \\
39\end{array}$ & $\begin{array}{l}3.23 \\
1.82\end{array}$ & $\begin{array}{l}1.05 \\
1.04\end{array}$ & 5.55 & .000 \\
\hline Medio ambiente & $\begin{array}{l}\text { Drama } \\
\text { Comedia }\end{array}$ & $\begin{array}{l}31 \\
39\end{array}$ & $\begin{array}{l}1.19 \\
1.69\end{array}$ & $\begin{array}{l}.477 \\
.922\end{array}$ & -2.73 & .008 \\
\hline Inmigración & $\begin{array}{l}\text { Drama } \\
\text { Comedia }\end{array}$ & $\begin{array}{l}31 \\
39\end{array}$ & $\begin{array}{l}2.26 \\
2.97\end{array}$ & $\begin{array}{l}1.41 \\
1.24\end{array}$ & -2.25 & .028 \\
\hline
\end{tabular}

Objetivo 1.3: Conocer la percepción de algunas variables conceptualizadas como prejuicios como son el rol narrativo, violencia como agresor, violencia como víctima, hábitos nocivos y temas de conversación en el personaje en el personaje inmigrante de las series de tv favoritas en función del origen de producción de la serie.

Según el origen de producción de las series se encontró que la mayor parte de la muestra $(55 \%)$ prefiere series de producción extranjera mientras que el resto $(45 \%)$ prefiere series de producción local.

Se realizaron pruebas $\mathrm{t}$ de student para verificar si las medias diferían respecto al origen de producción de las series preferidas por la muestra.

En el caso de la variable de rol narrativo del personaje inmigrante, en la prueba t se observan diferencias significativas en los roles de; protagonista y secundario protagónico. A diferencia de los roles antagonista, secundario no protagónico donde no hubo diferencias significativas en las medias como se puede ver en la tabla 8. Lo que indica que en las series de producción extranjera el personaje inmigrante fue percibido como protagonista y secundario protagónico. 


\section{TABLA 8}

Rol narrativo percibido en función del origen de producción de la serie

\begin{tabular}{|c|c|c|c|c|c|c|}
\hline Variable & Producción & $\mathrm{N}$ & Media & DT & $\mathrm{t}$ & $\mathrm{p}$ \\
\hline Rol Protagonista & $\begin{array}{l}\text { Local } \\
\text { Extranjera }\end{array}$ & $\begin{array}{l}32 \\
38\end{array}$ & $\begin{array}{l}2.44 \\
3.11\end{array}$ & $\begin{array}{l}1.29 \\
1.03\end{array}$ & -2.40 & .022 \\
\hline Rol Antagonista & $\begin{array}{l}\text { Local } \\
\text { Extranjera }\end{array}$ & $\begin{array}{l}32 \\
38\end{array}$ & $\begin{array}{l}2.34 \\
2.03\end{array}$ & $\begin{array}{l}1.23 \\
.944\end{array}$ & 1.12 & .239 \\
\hline Rol Secundario protagónico & $\begin{array}{l}\text { Local } \\
\text { Extranjera }\end{array}$ & $\begin{array}{l}32 \\
38\end{array}$ & $\begin{array}{l}3.00 \\
3.63\end{array}$ & $\begin{array}{l}1.01 \\
1.12\end{array}$ & -2.44 & .017 \\
\hline Rol Secundario no protagónico & $\begin{array}{l}\text { Local } \\
\text { Extranjera }\end{array}$ & $\begin{array}{l}32 \\
38\end{array}$ & $\begin{array}{l}2.47 \\
2.26\end{array}$ & $\begin{array}{l}1.16 \\
1.15\end{array}$ & .739 & .462 \\
\hline
\end{tabular}

En el caso de la variable de violencia, percibiendo al personaje inmigrante como agresor se hallaron diferencias significativas en el apartado de violencia fisica e intimidación como se puede ver en la tabla 9. Lo que indica que en las series extranjeras la muestra percibe que el personaje inmigrante hace más utilización de la violencia fisica y la intimidación.

TABLA 9

Percepción de violencia como agresor en función al origen de producción de la serie

\begin{tabular}{|c|c|c|c|c|c|c|}
\hline Variable & Producción & $\mathrm{N}$ & Media & DT & $\mathrm{t}$ & $\mathrm{p}$ \\
\hline Violencia física/agresor & $\begin{array}{l}\text { Local } \\
\text { Extranjera }\end{array}$ & $\begin{array}{l}32 \\
38\end{array}$ & $\begin{array}{l}1.53 \\
2.39\end{array}$ & $\begin{array}{l}.983 \\
1.24\end{array}$ & .045 & .002 \\
\hline Intimidación/agresor & $\begin{array}{l}\text { Local } \\
\text { Extranjera }\end{array}$ & $\begin{array}{l}32 \\
38\end{array}$ & $\begin{array}{l}1.81 \\
2.55\end{array}$ & $\begin{array}{l}1.23 \\
1.35\end{array}$ & .561 & .020 \\
\hline Daño a la propiedad/agresor & $\begin{array}{l}\text { Local } \\
\text { Extranjera }\end{array}$ & $\begin{array}{l}32 \\
38\end{array}$ & $\begin{array}{l}1.53 \\
1.82\end{array}$ & $\begin{array}{l}.803 \\
1.13\end{array}$ & .190 & .239 \\
\hline Violencia verbal/agresor & $\begin{array}{l}\text { Local } \\
\text { Extranjera }\end{array}$ & $\begin{array}{l}32 \\
38\end{array}$ & $\begin{array}{l}1.84 \\
2.34\end{array}$ & $\begin{array}{l}1.05 \\
1.43\end{array}$ & .008 & .108 \\
\hline Violencia sexual/agresor & $\begin{array}{l}\text { Local } \\
\text { Extranjera }\end{array}$ & $\begin{array}{l}32 \\
38\end{array}$ & $\begin{array}{l}1.28 \\
1.32\end{array}$ & $\begin{array}{l}.772 \\
1.01\end{array}$ & .171 & .875 \\
\hline
\end{tabular}

En el caso de la variable de la violencia siendo el personaje inmigrante víctima de esta se encontraron diferencias significativas en las medias que se puede ver en la tabla 10. Los datos hallados nos dicen que en las series de producción local el personaje inmigrante se percibe más como víctima de violencia fisica, intimidación y violencia verbal. 
TABLA 10

Percepción de violencia como víctima en función al origen de producción de la serie

\begin{tabular}{|l|l|c|c|c|c|c|}
\hline \multicolumn{1}{|c|}{ Variable } & \multicolumn{1}{|c|}{ Producción } & $\mathrm{N}$ & Media & $\mathrm{DT}$ & $\mathrm{t}$ & $\mathrm{p}$ \\
\hline \multirow{2}{*}{ Violencia física/víctima } & Local & 32 & 2.44 & 1.29 & \multirow{2}{*}{.002} & \multirow{2}{*}{.041} \\
& Extranjera & 38 & 1.87 & .906 & & \\
\hline \multirow{2}{*}{ Intimidación/víctima } & Local & 32 & 3.28 & 1.32 & \multirow{2}{*}{.062} & \multirow{2}{*}{.000} \\
\hline \multirow{2}{*}{ Daño a la propiedad/víctima } & Extranjera & 38 & 2.03 & 1.02 & & \\
& Local & 32 & 1.97 & 1.15 & \multirow{2}{*}{.002} & .013 \\
\hline \multirow{2}{*}{ Violencia verbal/víctima } & Extranjera & 38 & 1.39 & .718 & & \\
& Local & 32 & 3.84 & 1.24 & \multirow{2}{*}{.566} & .000 \\
\hline \multirow{2}{*}{ Violencia sexual/víctima } & Extranjera & 38 & 1.92 & 1.05 & & \\
& Local & 32 & 1.50 & 1.01 & \multirow{2}{*}{.000} & .118 \\
\hline
\end{tabular}

En cuanto a la variable hábitos nocivos se encontraron diferencias significativas en las medias del ítem de alcoholismo a favor de la producción extranjera como se observa en la tabla 11. En los demás apartados que componen esta variable como son fumador, adición a fármacos y adicción a sustancias ilegales no se encontraron diferencias significativas.

TABLA 11

Hábitos nocivos percibidos en función del origen de producción

\begin{tabular}{|l|l|c|c|c|c|c|}
\hline \multicolumn{1}{|c|}{ Variable } & \multicolumn{1}{|c|}{ Producción } & N & Media & DT & T & p \\
\hline \multirow{2}{*}{ Fumador } & Local & 32 & 1.31 & .859 & -.498 & .620 \\
& Extranjera & 38 & 1.42 & .948 & & \\
\hline \multirow{2}{*}{ Alcoholismo } & Local & 32 & 1.34 & .937 & -2.84 & .005 \\
& Extranjera & 38 & 2.05 & 1.14 & & \\
\hline \multirow{2}{*}{ Adicción a fármacos } & Local & 32 & 1.16 & .515 & \multirow{2}{*}{-1.54} & .108 \\
\hline \multirow{2}{*}{ Adicción a sustancias ilegales } & Extranjera & 38 & 1.47 & 1.05 & & \\
& Local & 32 & 1.19 & .471 & \multirow{2}{*}{-.900} & .371 \\
\hline
\end{tabular}

En la variable de temas conversación utilizada por el personaje inmigrante expuesto en la tabla 12, no se encontraron diferencias significativas en los siguientes temas: amor, violencia, sexo, trabajo, medio ambiente, salud, educación, familia, racismo e inmigración. En cambio se encontraron diferencias significativas en temas 
como la amistad, dinero, diferencias sociales y deporte. Por lo que se puede observar según este resultado es que en las series de producción extranjera la muestra percibe que el personaje inmigrante habla más sobre la amistad y deportes que sobre cualquier otro tema. Mientras que en las series local se percibe que hablan más de diferencias sociales y dinero.

TABLA 12

Temas de conversación recurrentes en función al origen de producción

\begin{tabular}{|l|l|c|c|c|c|c|}
\hline \multicolumn{1}{|c|}{ Variable } & \multicolumn{1}{|c|}{ Formato } & N & Media & DT & \multicolumn{1}{c|}{ t } & P \\
\hline \multirow{2}{*}{ Amistad } & Local & 31 & 3.03 & .933 & \multirow{2}{*}{.02} & \multirow{2}{*}{.000} \\
& Extranjera & 39 & 3.92 & .912 & & \\
\hline \multirow{2}{*}{ Dinero } & Local & 31 & 3.28 & 1.19 & \multirow{2}{*}{2.06} & .043 \\
& Extranjera & 39 & 2.68 & 1.21 & & \\
\hline \multirow{2}{*}{ Diferencias sociales } & Local & 31 & 3.22 & 1.33 & \multirow{2}{*}{2.22} & \multirow{2}{*}{.030} \\
& Extranjera & 39 & 2.53 & 1.26 & & \\
\hline \multirow{2}{*}{ Deporte } & Local & 31 & 1.38 & .660 & \multirow{2}{*}{-2.56} & .009 \\
& Extranjera & 39 & 2.03 & 1.30 & & \\
\hline
\end{tabular}

\section{Conclusiones y discusión}

Este estudio piloto es el punto de partida de una investigación más amplia que tiene como finalidad dar respuesta, a cómo perciben los jóvenes al inmigrante en las series de televisión. Cabe agregar que por naturaleza de los resultados y el tamaño de la muestra los datos de este estudio deben tomarse con cautela, ya que éstos no permiten que se pueda generalizar en estas variables. Con una metodología enteramente cuantitativa, se trata de analizar la percepción del sujeto de la muestra acerca de las características del inmigrante en las series de ficción.

El objetivo principal de este trabajo comprende varios objetivos específicos, respecto al primer objetivo, se ha hallado que el alumnado del grado educación infantil tiene una percepción mayor del hábito nocivo de fumar en el personaje inmigrante que el alumnado de comunicación. Asimismo los estudiantes de comunicación perciben al personaje inmigrante más como secundario no protagónico que es el menos importante dentro de una trama. Lo que tiene congruencia con lo hallado por Ruiz et al. (2007) ya que ellos explican que la figura del inmigrante en la ficción aparece de forma esporádica y marginada. Asimismo esto no corresponde con los hallazgos de Harwood y Anderson (2002) donde no se encontraron diferencias significativas respecto al rol de los personajes inmigrantes. Esto confirma que este fenó- 
meno debe continuar siendo estudiado, porque, aunque los estudios previos arrojen conclusiones similares en muchos aspectos difieren entre sí.

Sin embargo, cuando la serie tiene formato de drama la percepción de prejuicios del alumnado universitario es mayor que cuando es comedia. Aun así, este trabajo corrobora en un aspecto y difiere en otro con lo que concluyó Galán (2006). Aquel aspecto que difiere con la autora se refiere a las series dramáticas que ella analizó, donde se encontró a que los inmigrantes son utilizados en roles episódicos y ni siquiera secundarios. Mientras que en este estudio se ha encontrado que en las series dramáticas el personaje inmigrante fue percibido con roles de notoria importancia como protagonistas y antagonistas. Esta diferencia puede darse en cuanto al formato debido al mundo cambiante de la ficción televisiva que se va renovando rápidamente respecto a roles, tramas e inclusión (Bornaetxea, 2015). En este aspecto también es congruente con el trabajo de Lacalle (2008) en el cual la autora explica en su análisis que los inmigrantes tienen una representación positiva en las series dramáticas.

En cuanto a los datos en consonancia con Galán (2006; 2009), que de igual forma congruentes con Van Dijk (2007), se trata de que el personaje inmigrante es percibido más violento como agresor en todos los tipos de violencia excepto en la agresión sexual. En cambio en nuestro estudio solo se percibió al inmigrante como víctima en el caso de la violencia física. Por lo que el personaje ha sido percibido como agresor, y concuerda con que los temas de conversación recurrentes que más se percibe en el personaje inmigrante esté la violencia. De acuerdo con Mastro y Greenberg (2000) en el aspecto de enmarcar al personaje inmigrante en temas relativos a la violencia.

En esta línea Oliver y Raney (2011) especifican en su investigación que las series dramáticas estimulan la reflexión del espectador, pero lo que se refleja en este estudio es lo contrario, debido a que en las comedias el personaje inmigrante es percibido con temas de conversación recurrentes que evocan más a la reflexión como son el amor, el medio ambiente y la inmigración. Mientras que en el drama se reitera la percepción del personaje relacionado con la violencia por lo que el tema de conversación al que más recurre es la violencia como hemos dicho anteriormente.

En cuanto al origen de la producción se observa que los jóvenes de esta muestra prefieren ver series extranjeras en lugar de series locales no siendo congruentes estos datos con lo que establece Martínez-Serrano (2014). En cuanto rol narrativo del personaje, cuando la serie es extranjera se percibió que se le concedía más importancia al personaje inmigrante que en las series de producción local debido a los roles, ya que fueron percibidos como protagonistas y secundarios protagónicos. Esta percepción de los jóvenes en series de ficción de producción extranjera que son más consumidas, no coincide con el estudio de Igartua et al. (2013) y Ramos (2014), donde sitúan al inmigrante con tendencia a aparecer como villano/antagonista en el 
contenido. Aunque el rol de antagonista tiene su importancia dentro de la trama, el protagonista y el secundario protagónico muestran una mejor imagen que este rol y en la mayoría de casos se le da más importancia. Esta diferencia puede indicar un cambio respecto a la producción de las series y el tratamiento de la figura del inmigrante en las mismas, por lo que se podría profundizar en estudios posteriores.

Igualmente cuando la serie es de producción local el personaje inmigrante se percibe más como víctima de violencia siendo marginado como expone Galán (2006), exceptuando la violencia sexual. Mientras que en las series extranjeras fueron percibidos como agresores y no como víctimas. Asimismo, otro aspecto que llama la atención es que en series extranjeras se percibe al inmigrante como alcohólico. Es decir, que tanto en las series locales como extranjeras se percibe una espiral de violencia en torno al personaje inmigrante, acompañado en las producciones extranjeras por el alcoholismo lo que coincide en ciertos aspectos con las investigaciones realizadas por: Galán, (2009), Igartua, Barrios y Ortega, (2012), Mastro y Behm-Morawitz (2005), Ramos, Igartua, Frutos, Barrios, Mohedano y Naval (2014), Ramos (2014) que manifiestan que la figura del inmigrante está construida con prejuicios en las series de TV. Por lo cual, en este estudio los jóvenes han percibido actitudes de prejuicios en el personaje inmigrante en la mayoría de los casos.

Los temas de conversación recurrentes percibidos por la muestra en el personaje inmigrante, en las series locales fueron las diferencias sociales y el dinero, mientras que en las series extranjeras fueron percibidos en los inmigrantes temas como los deportes y la amistad. Se puede deducir que en cada pareja de temas tiene relación entre uno y otro lo que significa que lo que percibieron tiene una misma relación temática. Lo que evidencia que la producción de la serie se adapta al contexto social donde se emite; por lo que las series extranjeras pueden transmitir una realidad diferente respecto al contexto y por ello la realidad en cuanto a la inmigración estaría sesgada la mayor parte de las veces, por el consumo de estas producciones que tendrían prejuicios diferentes según el escenario donde se desarrolle la acción principal.

Es importante, señalar alguna limitación de este trabajo, recogido a través del auto informe, lo que implica la deseabilidad social que pueden tener las personas al momento de la cumplimentación de un cuestionario de esta naturaleza. Este tema puede abordarse de diferentes maneras en un futuro, según la popularidad de las series, así como con los formatos de las mismas, incluso como el enfoque de Adams (2018) y Arellano (2017), con un tipo etnia específica en el mundo de la ficción. También mezclando metodologías y técnicas como el análisis de contenido y los cuestionarios donde se podrían cruzar los datos para enriquecer más la investigación y de esta forma tener más claro el discurso mediático de la ficción televisiva sobre la inmigración en un determinado momento social, lo que ayudaría en la perspectiva de la educación mediática sobre temas como éste de gran relevancia social. 


\section{Agradecimientos}

Este estudio ha sido financiado por la Universidad del País Vasco (UPV/EHU). Referencia GIU 16/44.

\section{Referencias}

Adams, A. (2018). The Case of Rewards and Punishments: Analyzing Televisual Representations of Race and Gender in Two 21st-Century Criminal Drama Series.

Arellano, G. (2017). Latino Representation on Primetime Television in English and Spanish Media: A Framing Analysis.

Allport, G.W., Malfé, R., y Verón, E. (1968): «La naturaleza del prejuicio». Argentina: Eudeba.

Benavides, J.L., y Retis, J. (2005): «Miradas hacia Latinoamérica: La representación discursiva de los inmigrantes latinoamericanos en la prensa española y estadounidense». Palabra Clave, (13), 93-114.

Bermejo, J., y Núñez, M. (2008): «Valores y actitudes de los espectadores de series de ficción televisiva». Presentado en actas de I Congreso De La AE-IC Investigar La Comunicación. Santiago De Compostela, 35-55.

Bornaetxea, A.R. (2015): «Experiencias asociativas y colaborativas de diseño televisual. La televisión como herramienta de inclusión social». Obra Digital: Revista De Comunicación, (9), 12-27.

Carrasco Campos, A., y Universidad Miguel Hernández de Elche. (2010): «Teleseries: Géneros y formatos. Ensayo de definiciones». Miguel Hernández Communication Journal, (1), 174-200.

Cohen, J. (2001): «Defining identification: A theoretical look at the identification of audiences with media characters». Mass Communication \& Society, 4(3), 245-264.

Díaz, R.R. (2004): "Teoría de la Agenda-Setting aplicación a la enseñanza universitaria» (R.R. Díaz, Ed.) Alicante: OBETS Editorial.

Eberl, J., Meltzer, C.E., Heidenreich, T., Herrero, B., Theorin, N., Lind, F., Berganza. R., Boomgaarden. H. G., Schemer. C. y Strömbäck, J. (2018). The European media discourse on immigration and its effects: A literature review. Annals of the International Communication Association, 1-17.

Galán, E. (2006): «La representación de los inmigrantes en la ficción televisiva en España. Propuesta para un análisis de contenido. El comisario y Hospital Central». Revista Latina de Comunicación Social, 61. Recuperado el 13/01/2016 de http://www.ull.es/ publicaciones/latina/200608galan.htm

Galán, E. (2009): «Personajes, estereotipos y representaciones sociales. Una propuesta de estudio y análisis de la ficción televisiva». Revista ECO-Pós, 9(1), 58-81.

Gálvez, J.Á. (2009): «La representación mediática de la inmigración. Entre el encuadre y el estigma». Revista Del Ministerio De Trabajo e Inmigración, (80), 61-80. 
García de Castro, M. (2002): «La ficción televisiva popular: Una evolución de las series de televisión en España» (1st ed.) Barcelona: Gedisa.

Gerbner, G. (1996): «Fred Rogers and the significance of story». En M. Collins, y M.M. Kimmel, Mister Rogers Neighborhood (pp. 3-15). Pittsburgh, PA: University of Pittsburgh Press.

Giró, X., y Jarque, J.M. (2006). Prensa escrita e inmigración: Estudio sobre la opinión de los diarios sobre la inmigración procedente de fuera de la unión europea y sobre la cobertura informativa de conflictos destacados que tienen relación con ella (octubre 1999junio 2002). Zer-Revista De Estudios De Comunicación, 11(20)

Harwood, J., y Anderson, K. (2002). The presence and portrayal of social groups on primetime television. Communication Reports, 15(2), 81-97.

Hoekstra, S.J., Stos, A.N., Swendson, J.R., y Hoekstra, A.E. (2012): «Racial bias in dream content». Dreaming, 22(1), 10-17.

Igartua, J. (2013): «Attitudinal impact and cognitive channelling of immigration stereotypes through the news». Revista Latina De Comunicación Social, 68, 599-621.

Igartua, J.J. (2007): «Persuasión Narrativa». Alicante: Editorial Club Universitario.

Igartua, J.J. (2008): «Identificación con los personajes y persuasión incidental a través de la ficción cinematográfica». Escritos De Psicología, 2(1), 42-53.

Igartua, J.J., Barrios, Isabel M. y Ortega, Félix (2012): «Analysis of the image of immigration in prime time television fiction». Comunicación y Sociedad, XXV(2), 5-28.

Igartua, J.J., y Frutos, F.J. (2016). Procesos de recepción y efectos socio-cognitivos de películas sobre inmigración. El papel moderador del prejuicio hacia inmigrantes. Migraciones. Publicación Del Instituto Universitario De Estudios Sobre Migraciones, (40), 33-61.

Igartua, J.J., y Ramos, M.M. (2013): «Creación de personajes para la ficción televisiva: Influencia del tipo de personaje y del contexto de la acción narrativa». Espacios de Comunicación. IV Congreso Internacional De La Asociación Española De Investigación En Comunicación, 1101.

Igartua, J., Marcos, M., Alvidrez, S., y Piñeiro, V. (2013): «Ficción audiovisual, inmigración y prejuicio». Las Representaciones De Las Migraciones En Los Medios De Comunicación, 157-177.

Lacalle, C. (2008). El discurso televisivo sobre la inmigración: Ficción y construcción de identidad Omega.

López Vidales, N., González Aldea, P. y Medina-de-la-Viña, E. (2011): «Jóvenes y televisión en 2010: un cambio de hábitos». Zer, 30, 97-113.

López Vidales, N., Medina de la Viña, E., y González Aldea, P. (2013): «Los jóvenes españoles demandan una televisión con más ficción y entretenimiento». Revista Ámbitos De Comunicación, (22). Recuperado el 30/03/2016 de http://institucional.us.es/ ambitos/?p=398\#more-398

Mastro, D.E., y Behm-Morawitz, E. (2005). Latino representation on primetime television. Journalism \& Mass Communication Quarterly, 82(1), 110-130. 
Mastro, D.E., y Greenberg, B.S. (2000). The portrayal of racial minorities on prime time television. Journal of Broadcasting \& Electronic Media, 44(4), 690-703.

Monk-Turner, E., Heiserman, M., Johnson, C., Cotton, V., y Jackson, M. (2010). The portrayal of racial minorities on prime time television: A replication of the Mastro and Greenberg study a decade later. Studies in Popular Culture, 32(2), 101-114.

Martínez-Serrano, M.D.M (2014): «El perfil de consumo televisivo en estudiantes de grados en Educación/The television viewing habits in university students of grades in education». Revista Latinoamericana De Tecnología Educativa-RELATEC, 13(1), 101-109.

Martín, M.B., Muntada, M.C., Busquets, C.G., Pros, R.C., y Sáez, T.D. (2015): «Videojuegos, televisión y rendimiento académico en alumnos de primaria». Pixel-Bit.Revista De Medios y Educación, (46), 25-38.

Medrano, C.M., y Palacios Navarro, S. (2008): «Los hábitos y la dieta televisiva en distintas edades: Implicaciones educativas». Pixel-Bit.Revista De Medios y Educación, 31, 59-70.

Medrano, C.M., Martínez de Morentin, J.I., y Pindado, J. (2014): «Identificación con los personajes televisivos y valores percibidos por los y las adolescentes». Revista De Estudios De Juventud, (106), 31-48.

Menéndez, M.I.M. (2014): «Ponga una mujer en su vida: Análisis desde la perspectiva de género de las ficciones de TVE» mujeres» y» con dos tacones» (2005-2006)/Put a woman in your life: An analysis of the TVE series» mujeres» and» con dos tacones» (20052006) from a gender perspective». Área Abierta, 14(3), 61.

Montero, Y. (2005): «Estudio empírico sobre serial juvenil «Al salir de clase»: sobre la transmisión de valores a los adolescentes». Comunicar(25). Recuperado el 16/11/2015 de https://www.revistacomunicar.com/verpdf.php?numero $=25 \&$ articulo $=25-2005-070$

Montero, Y. (2006): "Televisión, Valores y Adolescencia». Barcelona: Gedisa.

Moreno, A. y Rodríguez, E. (2016): «Informe de juventud en España 2012». METAMORFOSIS, 112-118. Consultado de http://revistametamorfosis.es/index.php/ metamorfosis/article/view/15

Moyer-Gusé, E. (2008): «Toward a theory of entertainment persuasion: Explaining the persuasive effects of entertainment-education messages». Communication Theory, 18(3), 407-425.

Müller, F. (2009): «Entertaining anti-racism multicultural television drama, identification and perceptions of ethnic threat». Communications, 34(3), 239-256.

Muñiz, C., Serrano, F.J., Aguilera, R.E., y Rodríguez, A. (2013): «Estereotipos mediáticos o sociales. Influencia del consumo de televisión en el prejuicio detectado hacia los indígenas mexicanos». Global Media Journal México, 7(14), 93-113.

Oliver, M.B., y Raney, A.A. (2011): «Entertainment as pleasurable and meaningful: Identifying hedonic and eudaimonic motivations for entertainment consumption». Journal of Communication, 61(5), 984-1004.

Park, S. (2012): «Mediated intergroup contact: Concept explication, synthesis, and application». Mass Communication and Society, 15(1), 136-159. 
Prats, E., de Régil, M., Lombarte, S., Higueras, E., y Santamaria (2006): »El tratamiento televisivo de la diversidad cultural según los jóvenes de secundaria». Congreso internacional de educación intercultural. Formación del profesorado y práctica escolar Madrid. Recuperado de: https://www.academia.edu/2013850/El_tratamiento_televisivo_de_la_diversidad_ cultural_seg\%C3\%BAn_los_j\%C3\%B3venes_de_secundaria

Ramos, M.M. (2014): La Imagen De Los Inmigrantes En La Ficción Televisiva De Prime Time. Análisis y recomendaciones para los profesionales.

Ramos, M.M. (2014): «Principales estudios realizados sobre la representación de las minorías en la ficción televisiva». Chasqui Revista Latinoamericana De Comunicación, (126), 97108.

Ramos, M.M., Igartua, J.J., Esteban, F.J.F., Vicente, I.M.B., Mohedano, F.O., y Naval, V.P. (2014): «La representación de los personajes inmigrantes en los programas de ficción». Vivat Academia, 17(127), 43-71

Rovira, D.P., y Igartua, J.J. (1998): «Validez y fiabilidad de una escala de empatía e identificación con los personajes». Psicothema, 10(2), 423-436.

Ruiz, X., Ferrer, J., Obradors, M., Pujadas, E., y Pérez, O. (2007): «Los roles narrativos del inmigrante en la ficción televisiva. El caso de la ficción española de producción propia». En J.J, Igartua y C. Muñiz (Eds.), Comunicación, Inmigración y Sociedad (pp. 111126) Salamanca: Universidad de Salamanca.

Signorielli, N., Morgan, M., Gross, L., y Gerbner, G. (1996): «Crecer en la televisión: Perspectiva de aculturación». En J. Bryant y D. Zillman (Eds.), Los efectos de los medios de comunicación: Investigaciones y teorías (pp. 35-66) Barcelona: Paidós Ibérica.

Ugalde, L., Medrano, C.M., y Aierbe, A. (2012): «Identificación con personajes cinematográficos discapacitados y valores percibidos: Una investigación experimental con universitarios». Zer-Revista De Estudios De Comunicación, 17(32), 187-208.

Van Dijk, T. (2005): «Nuevo racismo y noticias». En M. Nash, R. Tello y N. Benach (Eds.), Inmigración, género y espacios urbanos. Los retos de la diversidad (pp.33-55) Barcelona, España: Edicions Bellaterra.

Van Dijk, T. (2007): «Discurso Racista». En J.J. Igartua, y C. Muñiz (Eds), Medios de comunicación, inmigración y sociedad (págs. 9-17). Salamanca: Ediciones Universidad de Salamanca. 